Z. Refaa ${ }^{1,2}$, M. Boutaous ${ }^{3}$, D. A. Siginer ${ }^{4,5}$

${ }^{1}$ Empa, Swiss Federal Laboratories for Materials Science and Technology, Dübendorf, Switzerland

${ }^{2}$ Competence Center Thermal Energy Storage Lucerne University of Applied Sciences and Arts, Horw, Switzerland

${ }^{3}$ Université de Lyon, CNRS, INSA-Lyon, CETHIL, UMR5008, Villeurbanne, France

${ }^{4}$ Centro de Investigacion en Creatividad y Educacion Superior y Departamento de Ingeniería Mecanica, Universidad de Santiago de Chile, Santiago, Chile

${ }^{5}$ Department of Applied Mathematics and Statistical Sciences, Department of Mechanical, Energy and Industrial Engineering, Botswana International University of Science and Technology, Palapye, Botswana

\title{
PLA Crystallization Kinetics and Morphology Development
}

This paper investigates the crystallization kinetics and morphology development of PLA. The transitory stages in the evolving flow-induced crystallization of PLA are identified and classified in terms of the overall crystallization kinetics and the crystalline morphologies. Under quiescent conditions, temperature governs the crystallization process and the slow crystallization kinetics of PLA is highlighted under these conditions, whereas under shearing conditions, the crystallization is highly enhanced due to the promotion of the nucleation mechanism. The enhancement of the crystallization implies also morphological modifications. Depending on the shear rate and the shearing time the microstructure changes dramatically: spherulitic microstructure, fine grained microstructure and oriented microstructure. For a specific shear rate, depending on the magnitude of the shearing time the microstructure assumes the following states: for low shearing time only an increase of the number of nuclei is observed (leading to fine grained microstructure), followed by a saturation of point-like nuclei, and for a relatively long shearing time (i.e. beyond a critical shearing time) the development of oriented structures looking like "shish-kebabs" is observed. The critical shearing time for the formation of oriented structures in PLA is determined as a function of the shear rate.

\section{Introduction}

Biobased polymers have gained more importance during the last decade with applications in various fields including medical sciences, packaging and agriculture. Among the available and the most promising biopolymers polylactide (PLA) stands out. This biopolymer is biodegradable aliphatic polyester produced from renewable resources such as corn. Nowadays PLA suppliers provide grades for several processes (injection mold-

* Mail address: M'hamed Boutaous, Université de Lyon, CNRS, INSA-Lyon, CETHIL, UMR5008, F-69621 Villeurbanne, France

E-mail: mhamed.boutaous@insa-lyon.fr ing, extrusion, rotomolding, etc). In spite of the progress made PLA still exhibits two major drawbacks: slow crystallization kinetics (leading to low mechanical performance) and low thermal stability under processing conditions. The latter is responsible of the PLA degradation (i.e. decrease of the molecular weight) at processing temperature. Considering the slow crystallization kinetics, the final part is almost amorphous. The improvement of the crystallization kinetics of PLA becomes a crucial task to enhance the final properties (dimensional stability, strength and flexural modulus...) and to extend its uses to other fields. To achieve this goal, one or both mechanisms of crystallization (i.e. nucleation and growth mechanisms) should be enhanced (Li and Huneault, 2007; Xiao et al., 2010; Nofar et al., 2013; Refaa et al., 2014; De Santis and Pantani, 2015). The incorporation of a nucleating agent to PLA provides heterogeneous nuclei (additional growing sites), leading to increasing crystallization rates. The addition of nucleating agent increases the crystallization rate (through additional heterogeneous nuclei) under isothermal and non-isothermal conditions leading to the increase of the crystallization temperature during cooling from the melt state. But, the effect of nucleating agent vanishes for relatively low cooling rate (Refaa, 2015; Courgneau et al., 2013). Hence, in some cases the enhancement of the growth rate is also required to achieve a significant crystallization rate with respect to processing conditions. Besides the slow crystallization kinetics, polylactide is a polymorphic polymer (Pan and Inoue, 2009). From the quiescent melt crystallization, two crystal forms can develop depending on the crystallization temperature; the $\alpha$ crystal form develops above $120^{\circ} \mathrm{C}$ and the $\delta$ crystal form develops below $100^{\circ} \mathrm{C}$ (Zhang et al., 2008). The two crystal forms exhibit different thermal properties, the enthalpy of $100 \% \alpha$ crystal form is $96 \pm 3 \mathrm{~J} / \mathrm{g}$ while its estimation for the $\alpha^{\prime}$ crystal form is $57 \pm 3 \mathrm{~J} / \mathrm{g}$ (Kalish et al., 2011).

With regards to processing conditions, quiescent crystallization is not representative and limited in describing the crystallization kinetics under flow conditions. Moreover, all the previous studies are conducted under quiescent conditions. Under polymers processing conditions, high cooling rates and high deformation rates are encountered. These severe thermome- 
chanical conditions affect particularly the kinetics of crystallization and the microstructure. Indeed, the shape of crystalline entities (spherulites or highly oriented structures "shish-kebabs") depends on the processing conditions. Below the thermodynamic equilibrium temperature, the flow is known to enhance the nucleation mechanism of semi-crystalline polymers, increasing the ability of the polymer to crystallize during processing. The growth rate of crystalline lamellae is also increased in the case of continuous shearing as demonstrated earlier by Monasse on a medium density polyethylene (Monasse, 1995). PLA is also sensitive to the presence of the flow. The application of a shear flow of short duration to PLA enhances the crystallization kinetics through supplementary nuclei (Zhong et al., 2013). The density of these nuclei increases with the shear rate and the shearing time; for long shearing times one can observe the saturation of point-like nuclei for a given shear rate. These observations also hold for other petrochemical based polymers for example iPP (Koscher and Fulchiron, 2002), MXD6 (Naudy et al., 2007).

Flow also affects the microstructure in addition to its effects on the kinetics of the process. Indeed, depending on the thermomechanical conditions and chemical properties of polymers (molecular weight, percentage of high molecular weight (HMW), branches), the impact on the crystallization and microstructure may vary. The increase of polymer molecular weight leads to more nuclei at the same shearing conditions as demonstrated by Koscher and Fulchiron, (2002) on isotactic polypropylene. Moreover, the increase of the high molecular weight content leads to the decrease of the specific mechanical work required for the formation of oriented structures "shishkebabs" (Mykhaylyk et al., 2008; Housmans et al., 2009).

This work is focused on the crystallization kinetics of PLA under quiescent as well as flow conditions given the importance of flow conditions during the processing of polymers concerning the kinetics of crystallization and the final microstructure. Both crystallization temperature and shear flow are considered with a particular attention to their impact on the overall crystallization kinetics and the morphology development of PLA. The transitory stages in flow-induced crystallization were shown and analyzed in terms of crystallization kinetics and microstructure. As far as we know, this is the first time where an overview of different transitions of flowinduced crystallization of PLA is reported and quantified providing more insight into the behavior of this biopolymer under real processing conditions. Finally a macro-kinetics model has been used for the prediction of nucleation density and the overall crystallization kinetics.

\section{Materials and Experimental Protocol}

The PLA used in this work is PLA 2003D (D-Isomer content is $4.25 \pm 0.55 \%$ ) supplied from Natureworks (Minnetonka, Minnesota, USA). The PLA was dried under vacuum to remove moisture and then extruded in a twin screw extruder, Haake Polylab OS PTW 16/25 (ThermoScientific, Waltham, Massachusetts, USA) with a barrel temperature of $180^{\circ} \mathrm{C}$ and a rotation speed of $25 \mathrm{~min}^{-1}$ over $3 \mathrm{~min}$. After extrusion, we determined the molecular weight average for PLA by size exclusion chromatography $M_{n}=74600 \mathrm{~g} / \mathrm{mol}$ and $M_{w}=97100 \mathrm{~g} / \mathrm{mol}$.

\subsection{Thermal Analysis}

Dynamic Scanning Calorimeter (DSC) is a powerful and accurate tool to investigate thermal transitions of polymeric materials; this apparatus is used in this work for the study of the isothermal melt crystallization of PLA. This study is conducted on a power compensation DSC (Pyris Diamond DSC, Perkin Elmer, Waltham, USA). A pellet of $5 \mathrm{mg}$ to $7 \mathrm{mg}$ weight undergoes the following thermal cycles: first, the sample is heated from room temperature up to $200{ }^{\circ} \mathrm{C}$, temperature higher than the equilibrium melting temperature (Refaa et al., 2017) and maintained over $2 \mathrm{~min}$ at this temperature in order to erase the thermomechanical history experienced by the polymer. The sample is then cooled at the rate of $30^{\circ} \mathrm{C} / \mathrm{min}$ to the desired crystallization temperature $T_{c}$ and maintained at $T_{c}$ until the end of the crystallization. For quantitative analysis we calculated the degree of transformation $\xi(\mathrm{t})$ by partial integration of the isothermal heat flow $\mathrm{Q}$ as follows:

$$
\xi(\mathrm{t})=\frac{\int_{\mathrm{t}_{\mathrm{i}}}^{\mathrm{t}} \mathrm{Q}(\mathrm{s}) \mathrm{ds}}{\int_{\mathrm{t}_{\mathrm{i}}}^{\mathrm{t}_{\infty}} \mathrm{Q}(\mathrm{s}) \mathrm{ds}},
$$

where $t_{i}$ and $t_{\infty}$ are the time when the isothermal conditions is reached and the end of the crystallization, respectively.

\subsection{Rheo-Optical Analysis}

The flow-induced crystallization of PLA is investigated using a hot stage shearing cell CSS450 (Linkam Scientific Instruments, London, UK) in conjunction with a polarized optical microscope Orthoplan (Leitz, Wetzlar, Germany), with one polarizer parallel to the flow direction. This setup allows in-situ observation of morphology development during experiments and provides quantitative information about the overall crystallization kinetics. Transmitted light intensity increases with the increase of crystalline phase and finally levels off at the end of the crystallization process. The overall crystallization can be investigated by monitoring the transmitted light intensity through the sample thickness using a camera QICAM-Fast 1394 (QImaging, Surrey, $\mathrm{BC}$, Canada). The results are presented in terms of the degree of transformation (i.e. the normalized transmitted light intensity):

$$
\xi(\mathrm{t})=\frac{\mathrm{I}(\mathrm{t})-\mathrm{I}_{\min }}{\mathrm{I}_{\max }-\mathrm{I}_{\min }},
$$

where $\mathrm{I}_{\min }$ and $\mathrm{I}_{\max }$ are the transmitted light intensity at the beginning and at the end of the crystallization process.

The shear flow is generated by rotating the lower circular plate while the higher plate is maintained fixed. Hence, a constant shear rate is imposed through the gap between the two parallel plates. The shear rate at the center of the window is controlled by the gap $d$ and the angular velocity $\omega\left(\dot{\gamma}=\frac{r_{0} \omega}{d}\right)$. The circular observation window is $2.8 \mathrm{~mm}$ in diameter and its position from the center is $r_{0}=7.5 \mathrm{~mm}$. We can estimate a relative error of $18.7 \%$ of the shear rate over the observation window. 
PLA films were placed in the hot stage and the following experimental protocol is applied, see Fig. 1. First, the sample was heated up to $200^{\circ} \mathrm{C}$ and maintained at this temperature for $2 \mathrm{~min}$ in order to erase its thermomechanical history. The gap between the two plates was reduced to $80 \mu \mathrm{m}$ after the melting of PLA (just prior to cooling step) and then the molten PLA was cooled down at the rate of $30^{\circ} \mathrm{C} / \mathrm{min}$ (maximum rate available) to the desired temperature of crystallization $\mathrm{T}_{\mathrm{c}}$. The cooling rate imposed on the sample is high enough to prevent the non-isothermal crystallization of PLA during cooling to the desired crystallization temperature. The choice of the crystallization temperature is a crucial step in order to obtain different types of crystalline entities (spherulites, shish-kebabs); indeed this temperature should be high enough to elucidate the impact of flow on the crystallization (kinetics and morphology); finally, the temperature was kept higher than $130^{\circ} \mathrm{C}$ (temperature where the $\alpha$ crystal form is found).

\section{Results and Discussion}

\subsection{Quiescent Crystallization}

The exothermal heat flow curves were used to deduce the relative crystallinity of PLA (i.e. degree of transformation) for each crystallization temperature. Figure $2 \mathrm{~A}$ shows the evolution of relative crystallinity versus time at different temperatures. Obviously, the faster crystallization is observed at $103^{\circ} \mathrm{C}$, this temperature represents the optimal thermal condi- tion for PLA crystallization. Nevertheless, the time needed to transform the whole sample is about $35 \mathrm{~min}$, which reflects slow crystallization kinetics. Moreover, the crystallization process takes more time to finish for low $\left(\mathrm{T}_{\mathrm{c}}<103^{\circ} \mathrm{C}\right)$ and high crystallization temperature $\left(\mathrm{T}_{\mathrm{c}}>103^{\circ} \mathrm{C}\right)$. As a result, during non-isothermal crystallization (e.g. cooling from the molten state), the crystallization process vanishes for relatively low cooling rates. This is clearly shown in several works in the literature (Refaa et al., 2017; Courgneau et al., 2013).

The time evolution of degree of transformation for each crystallization temperature can provide quantitative information about the crystallization process, and mainly about the crystallization rate and the shape of crystalline entities. The Avrami theory (Avrami, 1939) is usually adopted for the analysis of the crystallization. Indeed, the analysis with the Avrami theory leads to the crystallization rate, $\mathrm{k}$, and the Avrami index, $\mathrm{n}$, related to the nucleation mechanism and the shape of the growing crystalline entities. The logarithmic plot of the Avrami equation for each isothermal crystallization experiment is given in Fig. 2B, from which we can deduce the Avrami index (i.e. slope of the straight line) and the crystallization rate $\mathrm{k}(\mathrm{y}-$ intercept). The plot shows linear evolution of $\ln (-(1-\xi))$ versus $\ln (\mathrm{t})$, the Avrami index varies slightly around 2.9 (with 0.22 as standard deviation), which suggests a tridimensional growth of nuclei with instantaneous nucleation mechanism (i.e. heterogeneous nucleation).

The Avrami index for the two higher temperatures is slightly higher than 3 and the average Avrami index without these two temperatures is 2.8 . The average value over the whole range

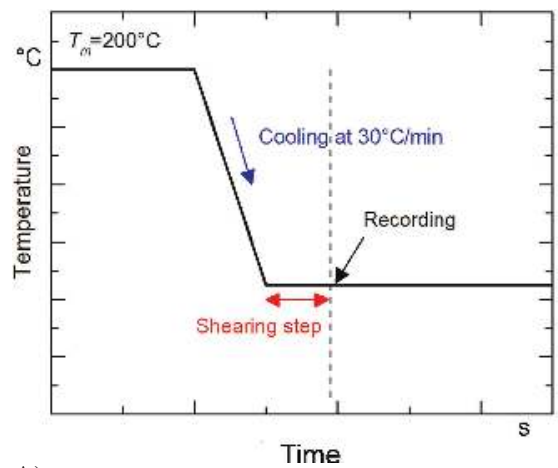

A)

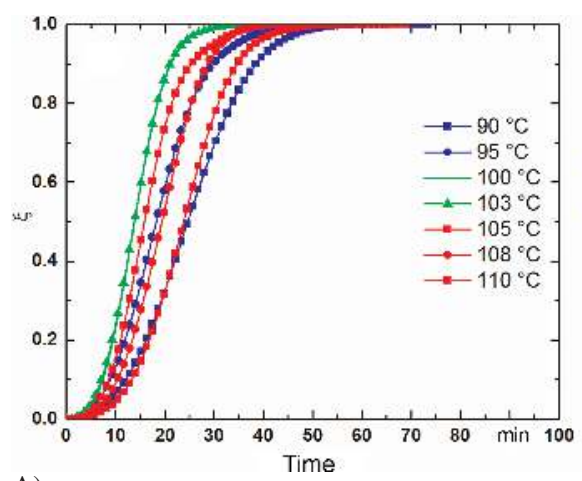

A)
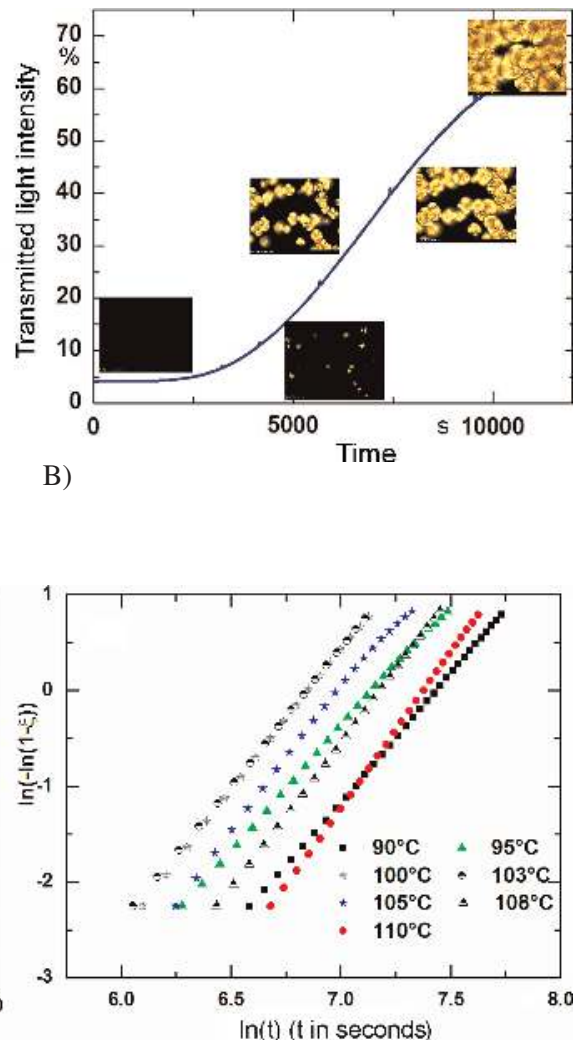

B)
Fig. 1. Temperature profile and shear proto$\operatorname{col}(A)$, profile of transmitted light intensity during isothermal crystallization (B)
Fig. 2. Degree of transformation versus time (A), logarithmic plot of the Avrami equation (B) 
of the investigated temperature is 2.9 , which suggests a tridimensional growth of crystalline entities with mainly heterogeneous nucleation. The slight change of the Avrami index for higher temperatures is interpreted as a change in the nucleation mechanism (from completely heterogeneous nucleation to the apparition of homogenous nucleation), but another interpretation can be given with regard to the polymorphism of PLA, since the studied range of temperature is considered as the transition zone from pure $\alpha$ crystal form to the $\alpha^{\prime}$-crystal form, as demonstrated in Refaa et al. (2015).

Flow is also known to largely impact on the kinetics and the microstructure of semi-crystalline thermoplastics (JaneschitzKriegl, 2010) in addition to the thermal dependence of the PLA crystallization. The following sections are dedicated to the crystallization kinetics and the morphology development of PLA, in order to emphasize the role of flow on the crystallization of PLA.

\subsection{Shear Induced Crystallization in PLA}

The crystallization temperature for shear flow experiments was chosen by considering two criteria: firstly the crystallization temperature should be high enough to bring out the impact of flow on the crystallization and secondly the crystallization should occur only in one crystal form zone. Finally, for the shear flow experiments $130^{\circ} \mathrm{C}$ is chosen, where only the $\alpha$ crystal form develops. At $130^{\circ} \mathrm{C}$, we applied a simple shear to the PLA melt and then monitored the crystallization and the morphology development, by measuring the transmitted light intensity through the crystallizing sample and by in-situ observation of the microstructure, respectively.

\subsubsection{Overall Crystallization and Microstructure}

The first experimental protocol is dedicated to the shear rate effects. We investigated the impact of different values of shear rate on overall crystallization kinetics and morphology development over a constant shearing time of $\mathrm{t}_{\mathrm{s}}=2 \mathrm{~s}$. The shear rate here can be seen as the ability of the flow to orient and/or to stretch macromolecules chains. We report in Fig. 3A the evolution of the degree of transformation versus time for several shear rates.
Shearing increases the ability of PLA to crystallize as can be seen from Fig. 3A. The transformation is accelerated after a shear rate of $2 \mathrm{~s}^{-1}$. For more clarity we define a characteristic time of crystallization, $\mathrm{t}_{1 / 2}$, in order to have an overview of the crystallization kinetics versus the shear rate. This time reflects the time needed to transform half of the sample (polymer melt) into the semi-crystalline state. The evolution of $t_{1 / 2}$ versus shear rate, given in Fig. 3B, shows a typical behavior found for other semi-crystalline polymers: after a critical shear rate, the flow becomes effective in the enhancement of the crystallization as shown by the dramatic decrease of the crystallization half time as shown in Fig. 3B. The change in the kinetics can be observed also in terms of the induced microstructure. Thanks to the rheo-optical setup, we had direct observation of the morphology development after the cessation of the shear flow. Figure 4 shows the impact of shear rate on the nucleation mechanism of PLA and the resulting crystalline microstructure.

The application of shear flow increases the number of nuclei (point-like nuclei), resulting in the development of small spherulites compared to quiescent conditions (coarse microstructure). Therefore a grained microstructure is obtained as the shear rate is increased. In the investigated range of shear rates, no oriented structures "shish-kebabs" were found. The formation of shish-kebabs is governed by two parameters of the shear flow: the shear rate and the shearing time (Mykhaylyk et al., 2008; Janeschitz-Kriegl, 2010). Indeed, for a given shear rate (high enough to ensure the stretching of macromolecular segments), there is a critical shearing time beyond which the development of fibrillar nuclei is observed. To describe the transition from point-like nucleation to thread-like nucleation both parameters of the shear flow are needed. For instance, Mykhaylyk et al. (2008) investigated the specific mechanical work ( $\mathrm{w}=\eta(\mathrm{T}, \dot{\gamma}) \dot{\gamma}^{2} \mathrm{t}_{\mathrm{s}}$ ) as criterion for the transition to fibrillar nucleation. It turned out that a critical amount of specific mechanical work is required for the formation of shish-kebabs. Furthermore, this critical amount of specific mechanical work is independent of the shear rate when it is higher than the inverse of the Rouse time of HMW molecules. Based on the previous discussion, a critical shear rate is required to ensure sufficient stretching conditions of macromolecular segments (van Meerveld et al., 2004) and a critical shearing time is necessary to obtain stable fibrillar nuclei. In our experiments, the shearing time of $2 \mathrm{~s}$ may be insufficient for the formation of stable fibrillar nuclei. To observe the transition to oriented structure

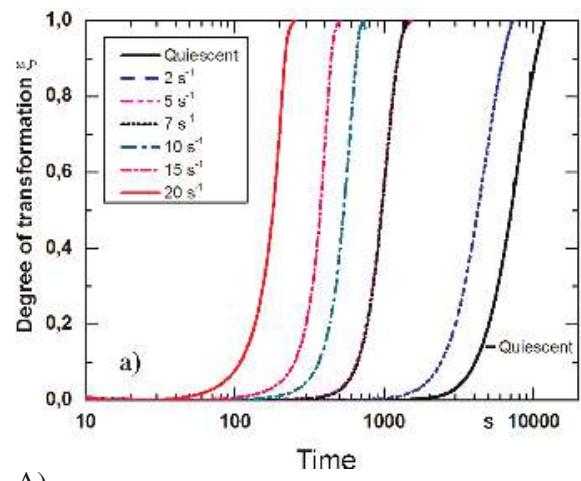

A)

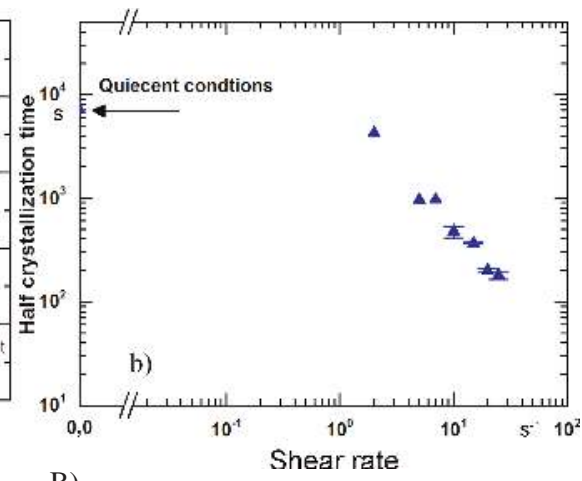

B)
Fig. 3. Evolution of degree of transformation versus time for different shear rates (A), half crystallization time versus shear rate at $130^{\circ} \mathrm{C}(\mathrm{B})$

Intern. Polymer Processing XXXIII (2018) 3 
the choice of the suitable shear rate is a crucial step to ensure the stretching of macromolecules and to increase the possibility for the development of fibrillar nuclei. Finally, for shear rates ranging between $5 \mathrm{~s}^{-1}$ and $25 \mathrm{~s}^{-1}$ we imposed several shearing times.

\subsubsection{Shearing Time and Formation of Oriented Structures}

In this section, the role of the shearing time on morphology development and crystallization kinetics of PLA is analyzed under isothermal conditions (i.e. $130^{\circ} \mathrm{C}$ ). In the following, for a given shear rate, the shearing time was varied in each experiment. The evolution of the half crystallization time versus the shearing time, for a shear rate of $10 \mathrm{~s}^{-1}$, is given in Fig. 5 .
For a given shear rate (i.e. $10 \mathrm{~s}^{-1}$ ), we were able to distinguish three different zones depending on the induced morphology and the overall crystallization kinetics of PLA. As shown in Fig. 5A a first acceleration of the overall crystallization kinetics (i.e. decrease of the half crystallization time $t_{1 / 2}$ ) is found, mainly due to the improvement of point-like nucleation, then a saturation of point-like nuclei can be seen from the evolution of the half crystallization time (plateau of the half crystallization time $t_{1 / 2}$ ). Finally for a relatively long shearing time, a second type of acceleration of the crystallization is observed.

The origins of the saturation-like behavior in the plateau zone (zone II) could also be due to the overshoot in the chain stretch, and could be linked to some rheological considerations in the PLA flow, as clearly explained in Roozemond and Peters (2013). More experiments should be done, with several PLA

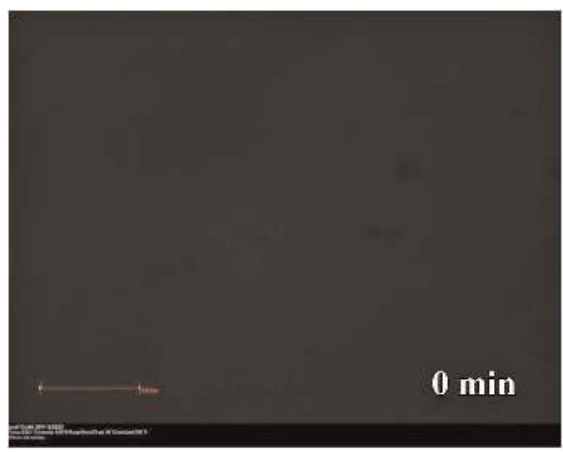

A)

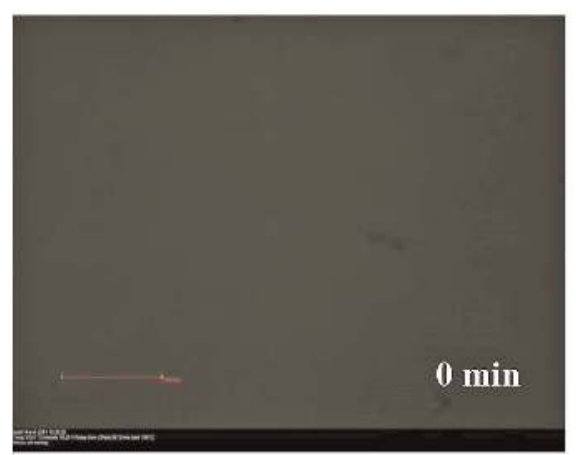

D)

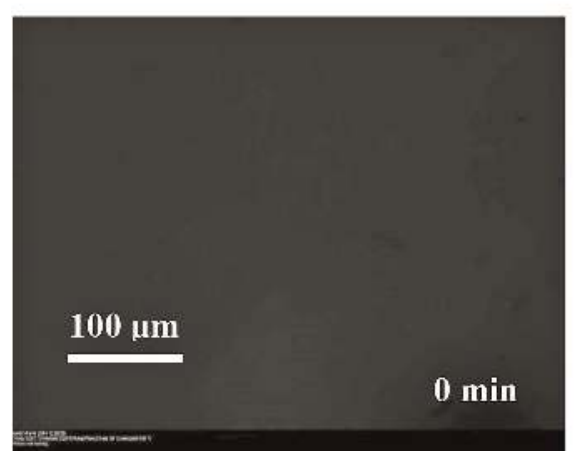

G)

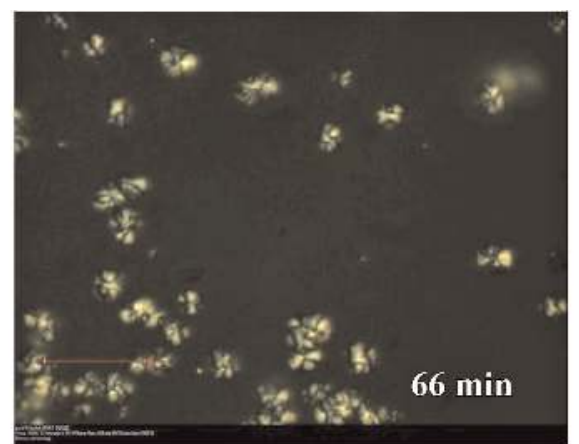

B)

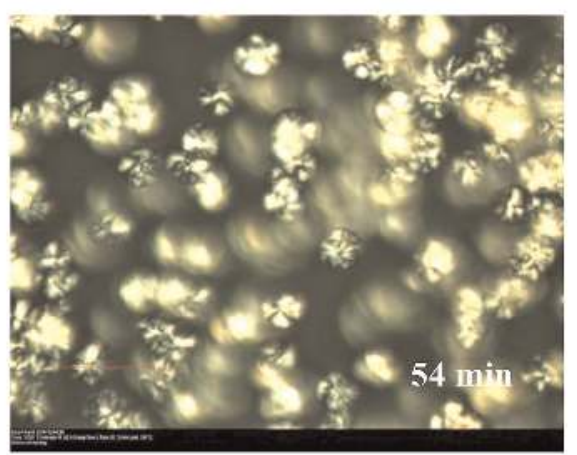

E)

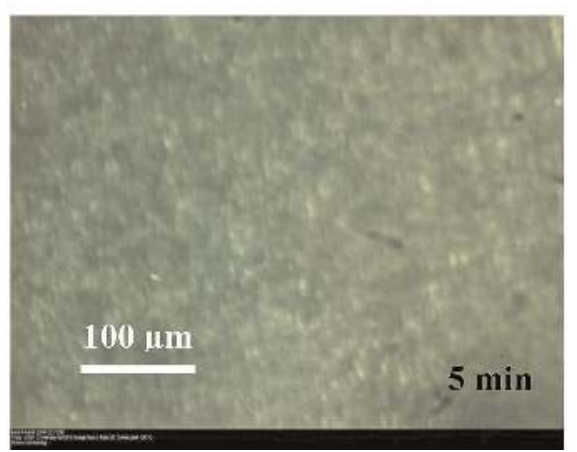

H)

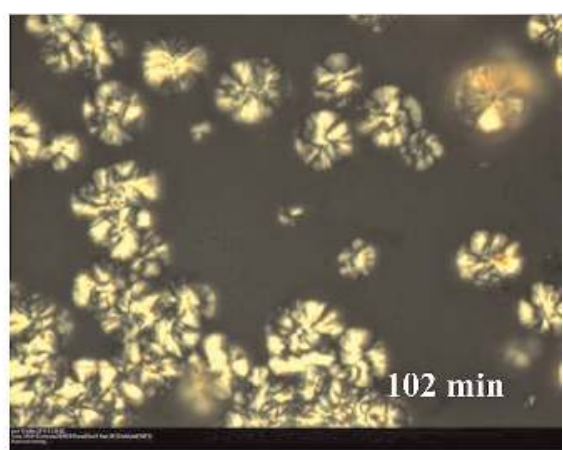

C)

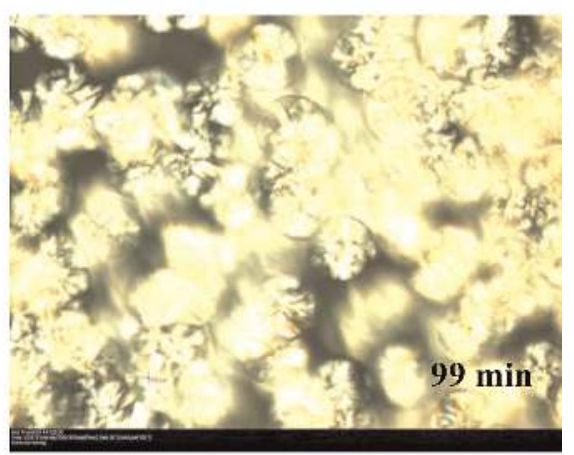

F)

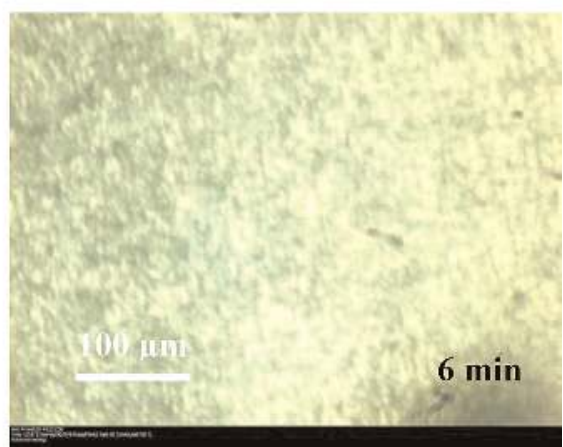

I)

Fig. 4. Nucleation and growth at: quiescent conditions $(A, B, C)$; shear rate of $2 s^{-1}(D, E, F)$ shear rate of $15 s^{-1}(I, J, K)$ 
with different molecular weight, in order to distinguish such phenomenon. In the current work, the saturation of point-like nuclei is considered, and that is not in conflict with that based on the overshoot in the chain strain.

This behavior of the half crystallization time versus shearing time was found also for an isotactic polypropylene(iPP) by Housmans et al. (2009), using a dynamic rheometer for the study of shear-induced crystallization. The Avrami analysis of the degree of transformation is given in Fig. 5B. Avrami index is located between 3 and 4 for zones I and II and decreases to reach a value between 1 and 2 in zone III. This behavior of the Avrami index reflects a morphological change from spherical growth of activated nuclei to a two dimensional growth characteristic of the growth of shish-kebabs (e.g. lateral growth of lamellae "kebabs" over fibrillar nuclei "shishs").

The polarized optical microscope observation supports the analysis based on the overall crystallization kinetics. Furthermore, it provides interesting information about the transition from point-like nucleation to fibrillar nucleation shish formation. At a shear rate $10 \mathrm{~s}^{-1}$, the following morphologies as function of the shearing time (Fig. 6) are distinguished: first, for low shearing time (zone I) only point-like nuclei develop as in Fig. 6A, then for mild shearing time (zone II), in the so called saturation region of point-like nuclei, we can observe in addition to small spherulites the development of intermediate structures known as row-nuclei or "cylindrulite", resulting from the alignment of several nuclei in the flow direction, see Fig. 6B. The latter are different from the well-known shish-ke- babs where the macromolecular segments are stretched in the flow direction forming the shish structure. Finally for high shearing times (zone III), we can observe the development of oriented structures "shish-kebabs". In Fig. 6C, we could not obtain clear shish-kebab structures due to the high density of fibrillar nuclei and relative high growth rate under these thermomechanical conditions. However, during the melting of the crystalline microstructure we can distinguish the presence of fibrillar structures having different melting behavior than spherulites, as shown in Fig. 7. Indeed, the light intensity decays around the spherulites while crossing the melting temperature, as can be seen in Fig. 7A, while in zone III (i.e. shish-kebabs development zone) a different melting behavior is observed - the light intensity vanishes around the oriented structures during the melting process, as can be seen in Fig. 7B.

The same experiment was conducted for other shear rates; the results are shown in Fig. 8. The evolution of $t_{1 / 2}$ with shearing time for each shear rate provides the dependence of the critical shearing time at that shear rate, for the transition from point-like nucleation to fibrillar nucleation. The critical shearing time for the formation of oriented structures of PLA decreases with the increase of the shear rate (Fig. 8A), as reported in the literature (Mykhaylyk et al., 2008; Housmans et al., 2009; Pantani et al., 2014). Furthermore, the density of oriented structures increases with the shear rate and shearing time. In order to quantify the transition from point-like nucleation to thread-like nucleation, the half crystallization time $\mathrm{t}_{1 / 2}$ versus strain was reported in Fig. 8B. For the investigated shear

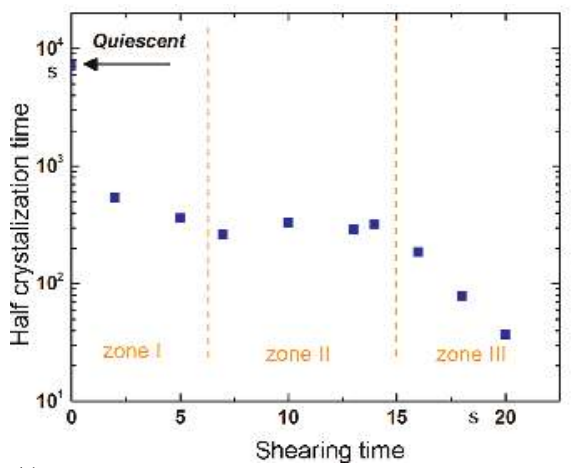

A)

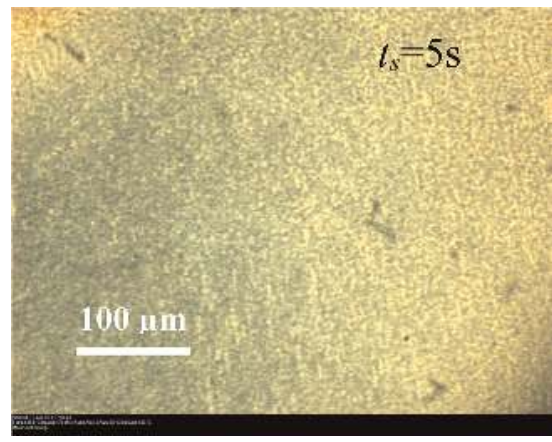

A)

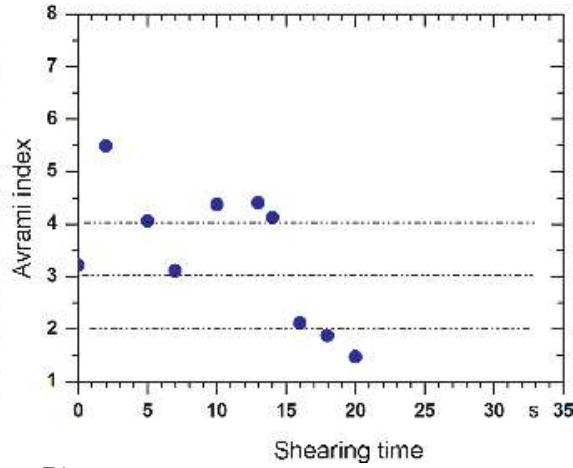

B)

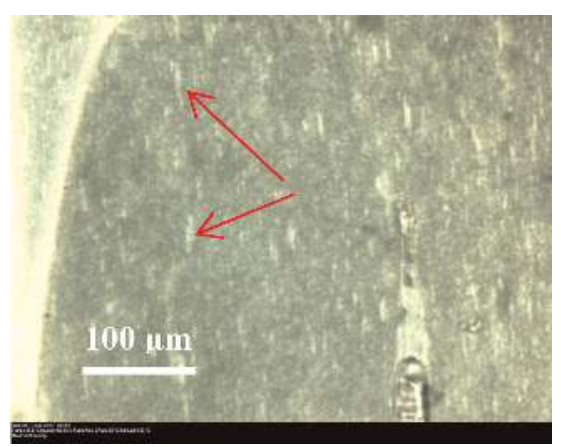

B)
Fig. 5. Half crystallization time versus shearing time (A), the Avrami index for different values of the shearing time at shear rate of $10 s^{-1}(B)$

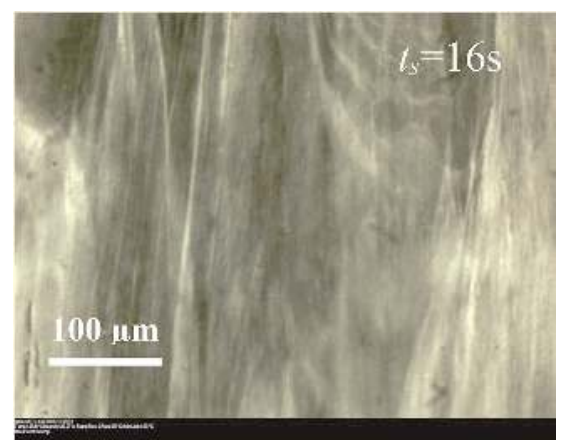

C)

Fig. 6. Typical microstructure for each characteristic zone $\left(T_{c}=130^{\circ} \mathrm{C}\right.$ and $\left.\gamma=10 \mathrm{~s}^{-1}\right), A: t_{s}=5 \mathrm{~s}, \mathrm{~B}: t_{s}=13 \mathrm{~s} ; \mathrm{C}: t_{s}=16 \mathrm{~s}$ 
rates, the transition to oriented structures was found to occur between a total strain between 100 and 160 (Fig. 8B).

\section{Flow-Induced Crystallization Modeling}

Flow effects on the crystallization can be reduced to its contribution to the nucleation mechanism, in the case of short term shearing (Koscher and Fulchiron, 2002). The number of flow- induced nuclei and their rate of formation depend on the shearing conditions. Indeed, the nucleation rate of shear induced nuclei depends on the state of orientation/stretching of macromolecular chains. The nucleation rate is usually linked directly to the shear rate, which is considered as the driven force for nucleation. Eder and coworkers (Liedauer et al., 1993) describe the rate of formation of point-like nuclei by a power law with an exponent of $2\left(\dot{\mathrm{N}} \propto \dot{\gamma}^{2}\right)$ which enables to describe the transition from spherultic morphology, M,M (coarse microstruc-

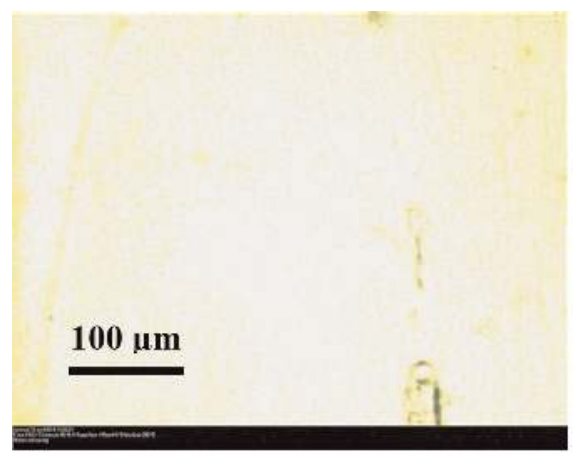

$\mathrm{T}=130,2^{\circ} \mathrm{C}$

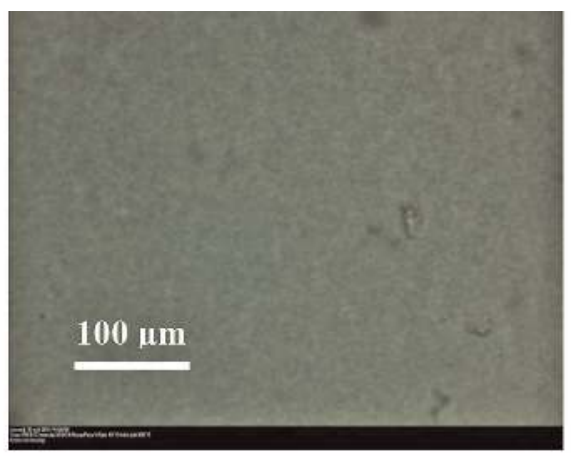

$\mathrm{T}=155,6^{\circ} \mathrm{C}$

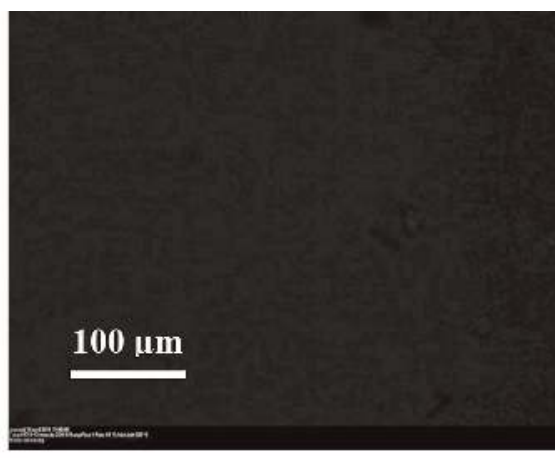

$\mathrm{T}=157^{\circ} \mathrm{C}$

A)

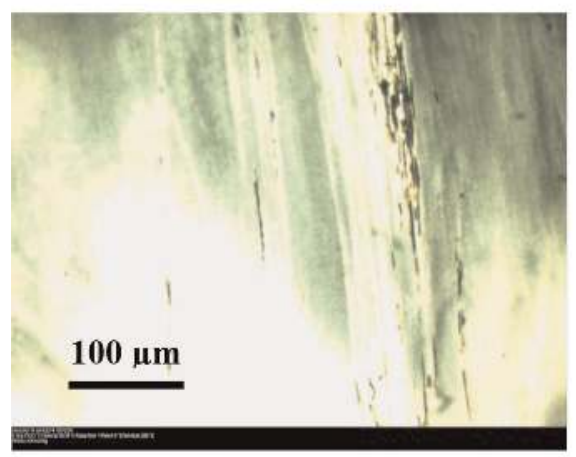

$\mathrm{T}=153,3^{\circ} \mathrm{C}$

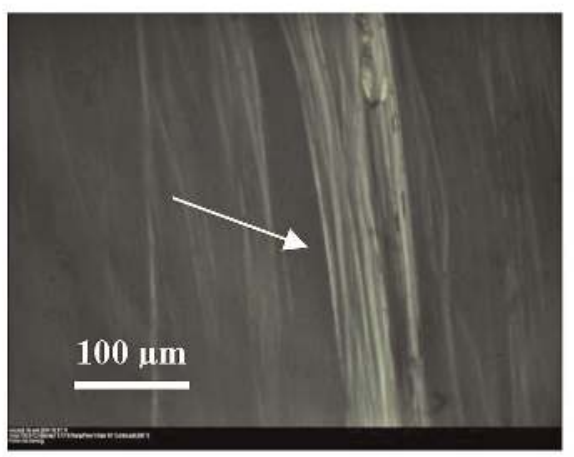

$\mathrm{T}=155,6^{\circ} \mathrm{C}$

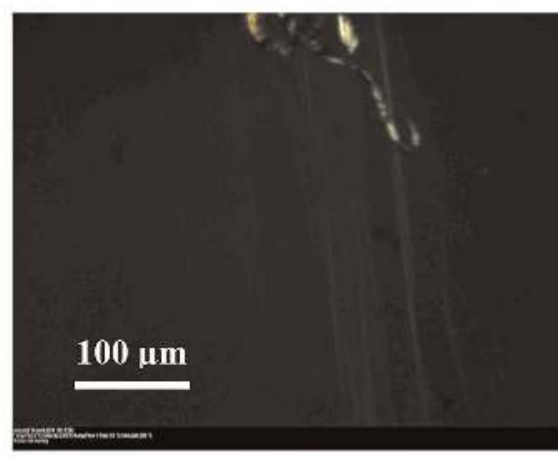

$\mathrm{T}=162^{\circ} \mathrm{C}$

B)

Fig. 7. Melting cycle $\left(\varphi=10^{\circ} \mathrm{C} / \mathrm{min}\right)$ after isothermal crystallization at $T_{c}=130^{\circ} \mathrm{C}$ and $\gamma=10 \mathrm{~s}^{-1}$, A) zone II : $t_{s}=13 \mathrm{~s}$, melting of spherulites, B) zone III : $t_{s}=20 \mathrm{~s}$, melting of shish-kebabs

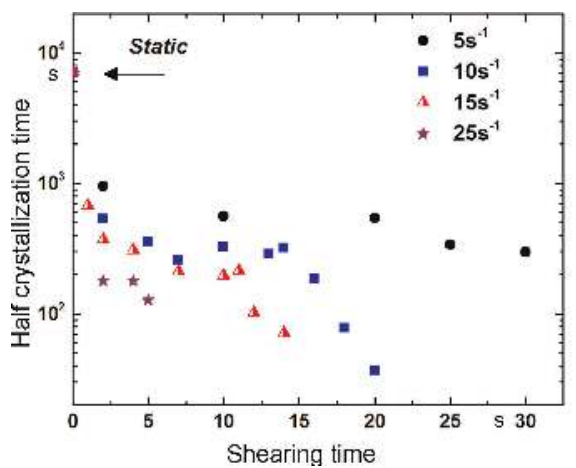

A)

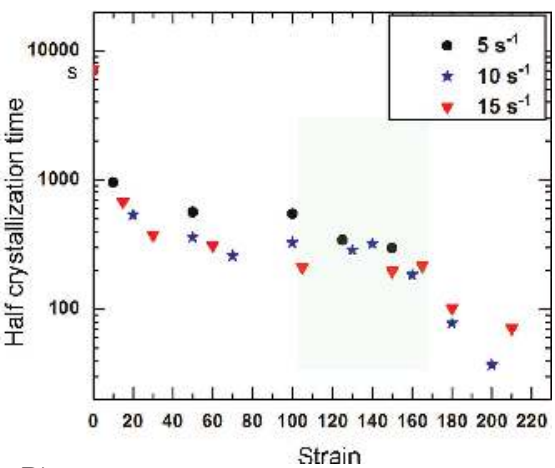

B)
Fig. 8. Half crystallization time versus shearing time (A) and versus strain $(B)$ for shear rate ranging from $5 s^{-1}$ to $25 s^{-1}\left(T_{c}=\right.$ $130^{\circ} \mathrm{C}$ ) 
ture), to fine grained structure due to the enhancement of pointlike nucleation. Furthermore, the transition from point-like nuclei to oriented structures "shish-kebabs" was found to scale with $\dot{\gamma}^{4} \mathrm{t}_{\mathrm{s}}=$ cte. In the same context, Janeschitz-Kriegl et al. (2003) investigated the specific mechanical work, w, provided to the polymer melt during shear treatment as the governing parameter of flow-induced crystallization and was linked to the number of point-like nuclei (for constant shear rate we have: $\mathrm{w}=\eta(\mathrm{T}, \dot{\gamma}) \dot{\gamma}^{2} \mathrm{t}_{\mathrm{s}}$ where $\eta$ the shear viscosity). Finally, for the modeling of flow-induced nucleation under isothermal conditions, the flow-induced nucleation rate depends only on the shear rate. Furthermore, for PLA with and without talc (i.e. nucleating agent) (Refaa et al., 2017) a linear evolution of $\ln \left(\dot{N}_{F}\right)$ versus $\ln (\dot{\gamma})$ was found. Finally, the following semi-empirical expression is valid in describing the dependence of flow-induced nucleation rate on the shear rate:

$\dot{\mathrm{N}}_{\mathrm{F}}=\mathrm{C}(\dot{\gamma})^{\mathrm{a}}$,

where a and $\mathrm{C}$ are two material parameters. In general these physical parameters of nucleation $\mathrm{C}$ and a depend on temperature and nucleation type. For polypropylene, Janeschitz-Kriegel (2010) reported the density of nuclei (thermally and flow induced nucleation) versus specific mechanical work, which is linked to shearing time and shear rate. The temperature has an influence on the slope and the intercept of the logarithmic plot of the density of nuclei versus specific work. Indeed, the slope decreases from 4 to 3 with the temperature decrease from $160^{\circ} \mathrm{C}$ to $140^{\circ} \mathrm{C}$, while the density of nuclei increases. Especially the "C" parameter is affected more by the temperature, while "a" is influenced more by the shear rate and the nucleation type.

In this paper, all our experiments were conducted under isothermal conditions, $\mathrm{Tc}=130^{\circ} \mathrm{C}$, hence, the modeling results concern only this isothermal temperature, and the identified parameters are taken as constant. Further measurements are needed, to extend the analysis to a larger range of temperatures.

Under these conditions, based on Avrami theory (Avrami, 1939) the flow-induced nucleation (FIN) rate can be obtained from the overall crystallization kinetics (i.e. half crystallization time versus shear rate at $\mathrm{t}_{\mathrm{s}}=2 \mathrm{~s}$ ) and the growth rate of spherulites (Naudy et al., 2007; Refaa et al., 2017; Refaa, 2015). The growth rate of spherulites is measured by following the radius of spherulites as a function of time at $130^{\circ} \mathrm{C}$. The growth rate was found to be $\mathrm{G}=0,36 \mu \mathrm{m} / \mathrm{min}$; this value is low compared to that for the pure PLLA. The growth rate of PLA depends on temperature and also is highly affected by molecular weight and optical purity (Tsuji et al., 2005). Finally, the flow-induced nucleation rate is identified from the evolution of the half-crystallization time versus shear rate conducted at a constant shearing time $\mathrm{t}_{\mathrm{s}}=2 \mathrm{~s}$, following the method proposed in Refaa et al. (2017). The two parameters a and $\mathrm{C}$ were identified by linear regression: $\mathrm{a}=3.1 ; \ln (\mathrm{C})=28.5$.

Using the identified flow-induced nucleation and the growth rate in the Avrami model, we can predict the overall crystallization under shearing conditions. Here we suggest the prediction of the half crystallization time versus shearing time. The simulation results are shown in Fig. 9; generally, the evolution of the half-crystallization time is well predicted by the proposed model. Indeed, the model predicts the first acceleration of the crystallization due to the enhancement of point like-nu-

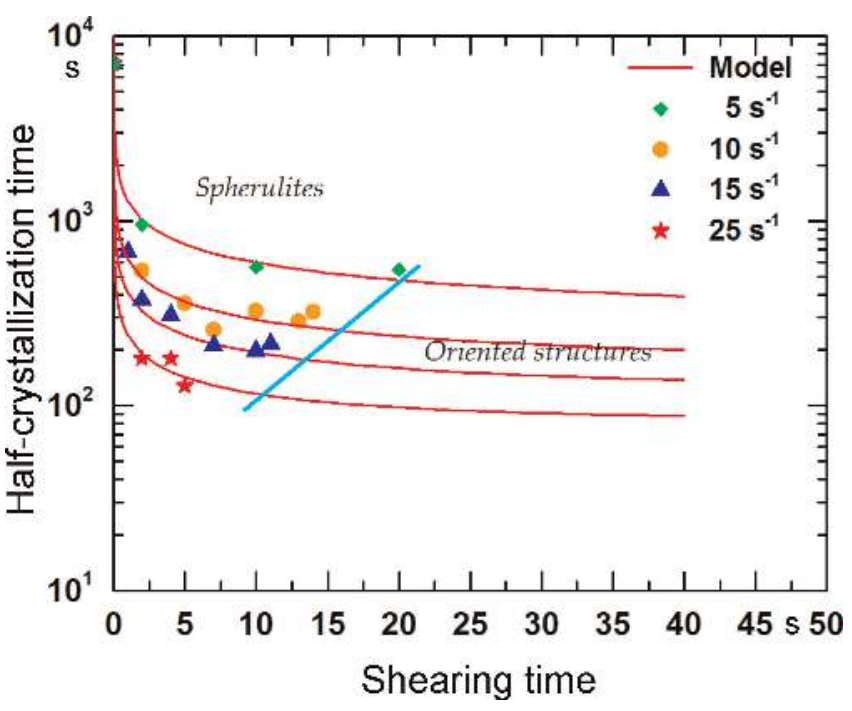

Fig. 9. Half-crystallization time versus shearing time at $T_{c}=130^{\circ} \mathrm{C}$ for shear rates $5 s^{-1} 10 s^{-1}, 15 s^{-1}, 25 s^{-1}$

cleation and captures also the saturation plateau related to the saturation of point-like nuclei and after which shish-kebabs are observed. However, a slight decrease of the half-crystallization time is predicted by the model instead of the saturation plateau. This difference may be related to an underestimation of the nucleation density activated under quiescent conditions. As shown in Fig. 9, the model doesn't predict the second acceleration of the crystallization, which is related to the development of oriented structures. In fact, the identified nucleation mechanism induced by the flow concerns only the "spherical nucleation". To this end, the model allowed us to assess the evolution of the overall crystallization kinetics versus shearing time; the enhancement and the saturation of point-like nuclei are well predicted.

\section{Conclusions}

Throughout this paper particular attention has been paid to the role of temperature and shear flow on the crystallization kinetics and the morphology development of PLA. On one hand, under quiescent conditions, the crystallization rate has a maximum around $103^{\circ} \mathrm{C}$. Beyond this temperature the crystallization time increases. Generally, the crystallization is too slow and an amorphous part is expected after cooling from the melt state. On the other hand, the flow plays a dramatic role in the enhancement of the crystallization kinetics even for a short shearing time of $2 \mathrm{~s}$. The impact on crystallization kinetics is due mainly to the enhancement of the nucleation mechanism as the micrographs show the tremendous effect of shear flow on the number of spherulites. Besides its influence on nucleation density, shear flow affects also the type of crystalline entities. Based on the overall crystallization kinetics we could distinguish three different behaviors (shearing time zone): a first acceleration related to the enhancement of point-like nucleation followed by a saturation of this type of nuclei; furthermore, we could distinguish in this zone the apparition of row- 
nuclei and finally a second acceleration observed for relatively high shearing time, attributed to the development of shish-kebabs. Finally, the enhancement of point-like nucleation and its saturation is predicted using the Avrami model.

\section{References}

Avrami, M., "Kinetics of Phase Change. I General Theory", J. Chem. Phys., 7,1103 (1939), DOI:10.1063/1.1750380

Courgneau, C., Ducruet, V., Avérous, L. Grenet, J. and Domenek, S., "Nonisothermal Crystallization Kinetics of Poly(lactide)-Effect of Plasticizers and Nucleating Agent", Polym. Eng. Sci., 53, 10851098 (2013), DOI:10.1002/pen.23357

Housmans, J. W., Steenbakkers, R. J., Roozemond, P. C., Peters, G. W. M. and Meijer, H. E. H., "Saturation of Pointlike Nuclei and the Transition to Oriented Structures in Flow-Induced Crystallization of Isotactic Polypropylene", Macromolecules, 42, 57285740 (2009), DOI:10.1021/ma802479c

Janeschitz-Kriegl, H., Ratajski, E. and Stadlbauer, M., "Flow as an Effective Promotor of Nucleation in Polymer Melts: A Quantitative Evaluation", Rheol. Acta, 42, 355-364 (2003), DOI:10.1007/s00397-002-0247-x

Janeschitz-Kriegl, H.: Crystallization Modalities in Polymer Melt Processing, Springer Vienna (2010), DOI:10.1007/978-3-211-87627-5

Kalish, J. P., Aou, K., Yang, X.-Z. and Hsu, S. L., "Spectroscopic and Thermal Analyses of $\alpha^{\prime}$ and $\alpha$ Crystalline Forms of Poly(1-lactic acid)", Polymer, 52, 814-821 (2011),

DOI:10.1016/j.polymer.2010.12.042

Koscher, E., Fulchiron, R., "Influence of Shear on Polypropylene Crystallization: Morphology Development and Kinetics", Polymer, 43, 6931-6942 (2002), DOI:10.1016/S0032-3861(02)00628-6

Li, H.-B., Huneault, M., "Effect of Nucleation and Plasticization on the Crystallization of Poly(lactic acid)", Polymer, 48, 6855-6866 (2007), DOI:10.1016/j.polymer.2007.09.020

Liedauer, S., Eder, G., Janeschitz-Kriegl, H., Jerschow, P., Geymayer, W. and Ingolic, E., "On the Kinetics of Shear Induced Crystallization in Polypropylene", Int. Polym. Proc., 8, 236-244 (1993), DOI:10.3139/217.930236

Meerveld, J. van, Peters, G. W. M. and Hütter, M., "Towards a Rheological Classification of Flow Induced Crystallization Experiments of Polymer Melts", Rheol. Acta, 44, 119-134 (2004), DOI:10.1007/s00397-004-0382-7

Monasse, B., "Nucleation and Anisotropic Crystalline Growth of Polyethylene under Shear", J. Mater. Sci., 30, 5002-5012 (1995), DOI:10.1007/BF01154515

Mykhaylyk, O. O., Chambon, P., Graham, R. S., Fairclough, J. P., Olmsted, P. D. and Ryan, A. J., "The Specific Work of Flow as a Criterion for Orientation in Polymer Crystallization", Macromolecules, 41, 1901 - 1904 (2008), DOI:10.1021/ma702603v

Naudy, S., David, L., Rochas, C. and Fulchiron, R., "Shear Induced Crystallization of Poly(m-xylylene adipamide) with and without Nucleating Additives", Polymer, 48, 3273 -3285 (2007), DOI:10.1016/j.polymer.2007.03.076

Nofar, M., Tabatabaei, A. and Park, C. B., "Effects of Nano-/MicroSized Additives on the Crystallization Behaviors of PLA and PLA/ CO2 Mixtures", Polymer, 54, 2382-2391 (2013), DOI:10.1016/j.polymer.2013.02.049

Pan, P.-J., Inoue, Y., "Polymorphism and Isomorphism in Biodegradable Polyesters", Prog. Polym. Sci., 34, 605-6040 (2009), DOI:10.1016/j.progpolymsci.2009.01.003
Pantani, R., Nappo, V., Santis, F. De, and Titomanlio, G., "Fibrillar Morphology in Shear-Induced Crystallization of Polypropylene", Macromol. Mater. Eng., 299, 1465-1473 (2014),

DOI:10.1002/mame.201400131

Refaa, Z., "Study of the PLA Crystallization: Effects of Nucleating Agent and Flow on the Crystallization Kinetics and Morphology Development", PhD Thesis, INSA de Lyon, France (2015)

Refaa, Z. Boutaous, M., Rousset, F., Fulchiron, R. Zinet, M., Xin, S. and Bourgin, P., "Crystallization Kinetics of Poly-(lactic acid) with and without Talc: Optical Microscopy and Calorimetric Analysis", AIP Conference Proceedings, 342-346 (2014), DOI:10.1063/1.4873796

Refaa, Z., Boutaous, M., Xin, S.-H. and Fulchiron, R., "Synergistic Effects of Shear Flow and Nucleating Agents on the Crystallization Mechanisms of Poly(lactic acid)", J. Polym. Res., 24, 18 (2017), DOI:10.1007/s10965-016-1179-y

Refaa, Z., Boutaous, M., Xin, S.-H. and Siginer, D. A., "Thermophysical Analysis and Modeling of the Crystallization and Melting Behavior of PLA with Talc", J. Therm. Anal. Calorim., 128, 687698 (2017), DOI:10.1007/s10973-016-5961-1

Roozemond, P. C., Peters, G. W. M., "Flow-Enhanced Nucleation of Poly(1-butene): Model Application to Short-Term and Continuous Shear and Extensional Flow", J. Rheol., 57, 1633 (2013), DOI: $10.1122 / 1.4821609$

Santis, F. De, Pantani, R., "Melt Compounding of Poly(lactic acid) and Talc: Assessment of Material Behavior during Processing and Resulting Crystallization", J. Polym. Res., 22, 242 (2015), DOI:10.1007/ s10965-015-0885-1

Tsuji, H., Tezuka, Y., Saha, S. K., Suzuki, M. and Itsuno, S., "Spherulite Growth of L-Lactide Copolymers: Effects of Tacticity and Comonomers", Polymer, 46, 4917-4927 (2005), DOI:10.1016/j.polymer.2005.03.069

Xiao, H. W., Li, P., Ren, X.-M., Jiang, T. and Yeh, J. T., "Isothermal Crystallization Kinetics and Crystal Structure of Poly(lactic acid): Effect of Triphenyl Phosphate and Talc", J. Appl. Polym. Sci., 118, 3558-3569 (2010), DOI:10.1002/app.32728

Zhang, J.-M., Tashiro, K., Tsuji, H. and Domb, A. J., "Disorder-to-Order Phase Transition and Multiple Melting Behavior of Poly(1 -lactide) Investigated by Simultaneous Measurements of WAXD and DSC", Macromolecules, 41, 1352-1357 (2008),

DOI:10.1021/ma0706071

Zhong, Y., Fang, H.-G., Zhang, Y.-Q., Wang, Z.-K., Yang, J.-J. and Wang, Z.-G., "Rheologically Determined Critical Shear Rates for Shear-Induced Nucleation Rate Enhancements of Poly(lactic acid)", ACS Sustainable Chem. Eng., 1, 663-672 (2013), DOI: $10.1021 / \mathrm{sc} 400040 \mathrm{~b}$

Date received: June 15, 2017

Date accepted: December 06, 2017

Bibliography

DOI 10.3139/217.3525

Intern. Polymer Processing

XXXIII (2018) 3; page 336-344

(c) Carl Hanser Verlag $\mathrm{GmbH} \&$ Co. KG

ISSN 0930-777X 